\title{
Chromohysteroscopy -A new technique for endometrial biopsy in Abnormal Uterine Bleeding (AUB) ${ }^{*}$
}

\author{
Nisha Singh", Bharti Singh \\ Department of Obstetrics \& Gynecology, King George Medical University, Lucknow, India \\ Email: "nisha.kgmc@gmail.com
}

Received 4 March 2013; revised 5 April 2013; accepted 13 April 2013

Copyright (C) 2013 Nisha Singh, Bharti Singh. This is an open access article distributed under the Creative Commons Attribution License, which permits unrestricted use, distribution, and reproduction in any medium, provided the original work is properly cited.

\begin{abstract}
Objective: To evaluate the role of chromohysteroscopy in improving diagnostic accuracy of endometrial biopsy in cases of AUB. Design: Cross sectional interventional study. Materials and Methods: This study was conducted on 60 women with AUB in Dept. of Obst. \& Gyne at King George Medical University, Lucknow over a period of one year. All cases underwent diagnostic hysteroscopy followed by chromohysteroscopy using $2 \%$ methylene blue dye. Hysteroscopic guided biopsy was taken from stained and unstained areas followed by an endometrial aspiration biopsy from whole uterine cavity. The histopathology results of three samples were compared and analyzed in relation with staining pattern and type of AUB. Data analysis was done on SPSS version 15 of windows 2007. Results: Out of 60 cases, 11cases were found to have non hormonal pathology after chromohysterosopic biopsy. Eight (72.72\%) cases were diagnosed by stained endometrial tissue, one $(9.09 \%)$ by unstained tissue and three $(27.27 \%)$ by endometrial aspiration. The diagnostic ability of stained tissue biopsy was significantly higher $(p=0.006)$ than unstained biopsy and endometrial aspiration. Conclusion: Chromohysteroscopy is a simple and effective technique for diagnosing endometrial pathology in cases of AUB.
\end{abstract}

Keywords: Endometrial Biopsy; Endometrial Aspiration; Hysteroscopy; Menstrual Abnormality; Methylene Blue Dye

\section{INTRODUCTION}

Abnormal Uterine Bleeding (AUB) is characterized by

\footnotetext{
"There is no conflict of interest in publication of this work. No commercial associations were involved.

${ }^{*}$ Corresponding author.
}

bleeding at unexpected time or by excessive or decreased flow at the time of expected menses. It is one of the most common clinical problems in gynecology practice. Almost $15 \%$ of outpatient cases and $25 \%$ of gyne-surgery cases present with AUB [1]. It is therefore important to understand the pathophysiology of AUB. Clinical history and examination help us to make a presumptive diagnosis based on the pattern of bleeding and presence of organic lesions. In absence of organic lesions, a battery of investigations is needed to establish the exact cause. Endometrial biopsy plays a pivotal role in diagnosis and treatment of such cases. Among the various methods of endometrial biopsy, hysteroscopic guided biopsy is considered gold standard. Yet, it is difficult to identify abnormal area during hysteroscopy except in presence of endometrial polyp or submucous fibroid.

"Chromoendoscopy" or "chromoscopy" refers to the topical application of stains or dyes at the time of endoscopy in an effort to enhance tissue characterization, differentiation and diagnosis. This technique identifies area that can be targeted for biopsy and is being used in gastrointestinal diseases with the help of methylene blue, Lugols's solution, Congo red and Indigo Caramine dyes [2]. The use of Methylene blue or other vital stains or dyes in the endometrium was not described, albeit clinically used, till the 1980s when it was used to distinguish endometrial tissue from fibrotic one in Asherman's syndrome [3]. Chromohysteroscopy is emerging as a new technique on the basis of chromoendoscopy. There is yet no consensus on the staining material and technique. The accuracy of diagnosis and its role in hysteroscopy needs further evaluation.

The present study was therefore planned to evaluate the diagnostic efficacy of chromohysteroscopy with methylene blue in cases of abnormal uterine bleeding.

\section{OBJECTIVE}

The primary objective was to detect difference in histo- 
logical diagnosis of stained and unstained endometrial tissue and compare each with histological diagnosis of endometrial aspiration. The secondary objective was to assess the diagnostic accuracy of chromohysteroscopy guided biopsy in cases of AUB.

\section{MATERIAL AND METHODS}

This interventional cross sectional study was carried out in the Department of Obstetrics and Gynecology at King George Medical University, Lucknow over a period of one year. Ethical clearance was taken from Institutional Ethical Committee. Sixty women with complaint of abnormal uterine bleeding were recruited after written informed consent. Detailed history and clinical examination were followed by investigations i.e. hemogram, thyroid profile, serum prolactin and transvaginal sonography. Women with bleeding disorder, thyroid disorder, hyperprolactinemia and fibroid were excluded.

Patients were taken up for chromohysteroscopy guided biopsy and endometrial aspiration between 20th - 25th day of menstrual cycle. Those with secondary amenorrhea or postmenopausal bleeding were taken up on first visit.

Initially, a diagnostic hysteroscopy was done with 4 $\mathrm{mm}$ diameter hysteroscope. Abnormal areas showing congestion, growth, fibrosis or adhesions were recorded. Hysteroscope was withdrawn. $2 \mathrm{ml}$ of $1 \%$ methylene blue dye was diluted with $8 \mathrm{ml}$ of normal saline. This 10 $\mathrm{ml}$ dye was pushed into the uterine cavity using a $5 \mathrm{~mm}$ Karman's cannula which is routinely used for endometrial biopsy. The authors did not instill the methylene blue through the operation channel of the hysteroscope because of the possible risk of permanent staining of the operation channel and improper sterilization thereafter.

Hysteroscope was reintroduced after waiting for five minutes to allow adequate exposure of endometrial lining to the dye. Staining pattern of endometrium and abnormal areas was recorded. Hysteroscopic biopsy forceps was introduced through side channel and endometrial biopsy was taken from stained \& unstained areas. Hysteroscope was withdrawn. Endometrial aspiration was done with another $5 \mathrm{~mm}$ Karman's cannula and 20 cc syringe. All three tissue samples were sent for histopathological evaluation.

Histopathology results were classified as normal, hormonal disturbance or endometrial pathology after studying all three biopsy results. Hormonal disturbance was diagnosed if histology did not correlate with the phase of menstrual cycle. Patients were diagnosed of endometritis when histological reports showed chronic inflammation.

Data record and analysis-All clinical and histological data was recorded on MS word excel sheet and then analyzed using SPSS 15 of MS windows 2007.

\section{RESULTS}

Table 1 shows age distribution of the 60 cases recruited. The mean age of these women was $33.40 \pm 8.80$ years and most cases (83.4\%) were in the age group of $21-40$ years. Out of 60 women, $36(60 \%)$ women had normal while $24(40 \%)$ had abnormal hysteroscopic findings. Histopathological results of endometrial biopsy of all cases are shown in Table 2. Out of 11 cases showing endometrial pathology in one or more of the three samples, five were those with normal hysteroscopy findings. The endometrial pathologies in these five cases with normal hysteroscopy were simple glandular hyperplasia [2], tubercular endometritis [1], and chronic inflammation [2].

Out of 4 cases with chronic inflammation, 2 showed staining on all walls and two showed patchy staining on chromohysteroscopy.

Table 3 shows that out of 11 endometrial pathologies, eight $(72.72 \%)$ were detected by stained tissue biopsy, three $(27.27 \%)$ by endometrial aspiration and only one $(9.09 \%)$ by unstained tissue biopsy. The diagnostic abil-

Table 1. Distribution of subjects according to age group $(\mathrm{n}=$ 60).

\begin{tabular}{cccc}
\hline S. No. & Age group (Years) & No. of subjects & Percentage \\
\hline 1. & $21-30$ & 28 & $46.7 \%$ \\
2. & $31-40$ & 22 & $36.7 \%$ \\
3. & $41-50$ & 7 & $11.7 \%$ \\
4. & $51-60$ & 2 & $3.33 \%$ \\
5. & $61-70$ & 1 & $1.67 \%$ \\
\hline
\end{tabular}

Table 2. Distribution of cases according to histopathology results $(n=60)$.

\begin{tabular}{cccc}
\hline & HPE REPORT & Number of cases & Percentage \\
\hline 1. & Normal & 29 & $48.33 \%$ \\
2. & Hormonal disturbance & 19 & $31.66 \%$ \\
3. & Endometrial pathology & & \\
a & Chronic inflammation & 4 & $6.66 \%$ \\
b & Tubercular endometritis & 3 & $5 \%$ \\
c & Endometrial hyperplasia & 3 & $5 \%$ \\
d & Endometrial carcinoma & 1 & $1.6 \%$ \\
4. & Inconclusive & 1 & $1.6 \%$ \\
\hline
\end{tabular}

Table 3. Endometrial pathology diagnosed from different methods of biopsy $(\mathrm{n}=11)$.

\begin{tabular}{ccc}
\hline Method of diagnosis & Number of cases & \% \\
Stained tissue biopsy & 8 & 72.72 \\
Endometrial aspiration & 3 & 27.27 \\
Unstained tissue biopsy & 1 & 9.09 \\
\hline$\chi^{2}=10.214 ; \mathrm{p}=0.006$. & &
\end{tabular}


ity of stained tissue biopsy was significantly higher $(\mathrm{p}=$ 0.006) than both endometrial aspiration and unstained biopsy.

Table 4 shows the comparison of histopathology results of three endometrial samples of each of the eight women whose disease was picked up only in the stained tissue sample.

\section{DISCUSSION}

Lim et al. (2002) [4] \& Allameh et al. (2007) [5] performed hysteroscopy in women with abnormal uterine bleeding and found that the mean age was 42 years and 38.6 years respectively. Hodo Mansoura et al. (2011) [6] evaluated the role of chromohysteroscopy in diagnosis of endometritis in absence of macroscopic pathology on transvaginal sonography and hysteroscopy in 57 cases of dysfunction uterine bleeding, unexplained infertility, recurrent first trimester abortion and failed IUI. The women aged between 24 to 38 years. In the present study, the mean age is 33.4 years. The lower mean age is attributable to the fact that all menstrual abnormalities were included in contrast with the above studies that included only menorrhagia, polymenorrhagia and metrorrhagia cases.

Methylene blue is an absorptive stain that is preferentially absorbed across epithelial cell membranes. Kucuk et al. [7,8] and Hodo Mansoura et al. [9] found that inflammatory cells had a higher uptake of methylene blue helping in diagnosis of unexplained endometritis. Role of methylene blue staining in discriminating malignant and non malignant tissue is not well defined. Meatsumato et al. [10] found that dysplastic cells predominantly take up methylene blue. Kucuk et al. [11] used chromohysteroscopy in 22 cases of postmenopausal bleeding but it did not increase the diagnosis of endometrial carcinoma. In our study, the single case of endometrial carcinoma did not show any staining of endometrium. The authors could not evaluate the role of chromohysteroscopy in distinguishing malignant from non malignant tissue because only one case of CA endometrium was diagnosed.

Tansu Kucuk et al. (2008) [7] performed chromohysteroscopy for evaluation of endometrium in 63 cases of recurrent IVF failure. Focal dark staining area was ob- served in 22 cases. Histopathology showed endometritis in nine of them. Thus chromohysteroscopy had sensitivity of $69.2 \%$, specificity of $74 \%$, positive predictive value of $40.9 \%$ and negative predictive value of $90.2 \%$ for endometritis. Kucuk T, Devici S et al. (2008) [8] also performed chromohysteroscopy in 34 cases of recurrent miscarriage. Out of 19 cases with focal dark staining, 10 were diagnosed as endometritis by biopsy. Hodo Mansoura et al. (2011) [9] reported that chromohysteroscopy had $70 \%$ sensitivity, $80.8 \%$, specificity, $43.7 \%$ PPV and 92.6\% NPV for diagnosis of chronic endometritis.

In the present study, any staining was considered positive rather than subjectively dividing it into light and dark staining. Staining was therefore recorded in $90 \%$ of cases.

It is generally presumed that endometrial tissue will take the stain while the fibrotic tissue will not. Our study included 12 cases of fibrous bands and adhesions. Staining was present in 7 of these cases indicating presence of focal endometrial tissue. Staining was absent in 5 cases and biopsy could not be taken in 3 of them due to presence of tough tissue. The other two unstained samples showed proliferative histology and mixed histology respectively. Thus, our findings suggest that contrary to general assumption, fibrotic bands may take up stains and may have some endometrial tissue. The unstained tissue biopsies also showed variety of histological findings according to phase of menstrual cycle suggesting the presence of endometrium in unstained areas (Table 4).

29 cases with normal histopathologic reports (48.33\%) and 19 cases with hormonal disturbance (31.66\%) did not show discrepancy in the three biopsy samples taken from each case. In contrast, evaluation of the biopsy reports of 11 cases with endometrial pathology (18.2\%) showed that eight of these were picked up only in the biopsy from stained area. The diagnostic accuracy of stained tissue biopsy to detect endometrial pathology was higher than that of unstained tissue biopsy and endometrial aspiration with a statistically significant difference $(p=0.006)$. Out of 11 cases with endometrial pathology, five (45.4\%) had no abnormality detected on plain hysteroscopy. This shows that chromohysteroscopy

Table 4. Histopathology results of different bipopsy samples in cases with disease diagnosed in stained tissue biopsy ( $\mathrm{n}=8)$.

\begin{tabular}{|c|c|c|c|}
\hline Menstrual abnormality & Stained tissue biopsy & Unstained tissue biopsy & Endometrial aspiration biopsy \\
\hline Polymenorrhagia & Simple glandular hyperplasia & Proliferative & Proliferative \\
\hline Secondary amenorrhea & Tubercular endometritis & Epitheloids cells with langerhans cells & Secretory endometrium \\
\hline Oligomenorrhea & Tubercular endometritis & Broken bits of endometrial gland & Arias stella reaction \\
\hline Menorrhagia & Simple glandular hyperplasia & Secretory & Secretory \\
\hline Menorrhagia & Chronic inflammation & Secretory & Secretory \\
\hline Menorrhagia & Chronic inflammation & Non secretory & Endometrial hyperplasia \\
\hline Hypomenorrhea & Chronic inflammatory & Broken bits of endometrial gland & Broken bits of endometrial glands \\
\hline
\end{tabular}


added to the diagnostic accuracy of hysteroscopy in 45.5\% cases which is a great advantage.

It is therefore, concluded from this study that chromo hysteroscopy is a useful technique which can improve the diagnostic accuracy of endometrial biopsy especially in cases with normal hysteroscopy. It is a cost effective technique without any side effects that may be tried in all cases of AUB.

The limitation of the study was the small number of cases of postmenopausal bleeding and hence its inability to comment on role of chromohysteroscopy in detecting endometrial cancer. Another study may be planned among women with postmenopausal bleeding to evaluate usefulness of chromohysteroscopy in diagnosis of endometrial malignancy.

\section{ACKNOWLEDGEMENTS}

This study was conceptualized and conducted by Dr Nisha Singh. Dr Bharti Singh assisted in all cases, recorded and analyzed the data. Dr. Nisha Singh prepared the final manuscript.

\section{REFERENCES}

[1] Shayder, J.M. (2000) Pathophysioogy of abnormal uterine bleeding. Obstetrics \& Gynecology Clinics of North America, 27, 219-234. doi:10.1016/S0889-8545(00)80017-0

[2] Ragunath, K., Krasner, N., Raman, V.S., et al. (2003) A randomized, prospective cross-over trial comparing methylene blue-directed biopsy and conventional random biopsy for detecting intestinal metaplasia and dysplasia in Barrett's esophagus. Endoscopy, 35, 998-1003. doi:10.1055/s-2003-44599

[3] Valle, R.F. and Sciarra, J.J. (1988) Intrauterine adhesions: Hysteroscopic diagnosis, classification, treatment, and re- productive outcome. American Journal of Obstetrics \& Gynecology, 158, 1459-1470

[4] Lim, C.H., Rotimi, O., Dexter, S.P.L., et al. (2006) Randomized crossover study that used methylene blue or random 4-quadrant biopsy for the diagnosis of dysplasia in Barrett's esophagus. Gastrointestinal Endoscopy, 64, 159. doi:10.1016/j.gie.2005.07.025

[5] Stevens, P.D., Lightdale, C.J., Green, P.H., et al. (1994) Combined magnification endoscopy with chromoendoscopy for the evaluation of Barrett's esophagus. Gastrointestinal Endoscopy, 40, 747-749.

[6] Gossner, L., Pech, O., May, A., et al. (2006) Comparison of methylene blue-directed biopsies and four-quadrant biopsies in the detection of high-grade intraepithelial neoplasia and early cancer in Barrett's oesophagus. Digestive and Liver Disease, 38, 724-729. doi:10.1016/j.dld.2006.05.025

[7] Kucuk, T. and Safali, M. (2008) Chromohysteroscopy for evaluation of endometrium in recurrent in vitro fertilization failure. Journal of Assisted Reproduction and Genetics, 25, 79-82.

[8] Kucuk, T. (2008) Chromohysteroscopy and quot for evaluation of endometrium in recurrent miscarriage. Clinical \& Experimental Obstetrics \& Gynecology, 35, 133-136.

[9] Mansoura, H. and Mohamed, A. (2011) value of endometrial dyeing in diagnosis of endometritis in absence of macroscopic abnormalities during hysteroscopy. Middle East Fertility Society Journal, 16, 83-86.

[10] Matsumotu, T., Nakamura, S., Jo, Y., et al. (2003) Chromoscopy might improve diagnostic accuracy in cancer surveillance for ulcerative colitis. The American Journal of Gastroenterology, 124, 880-888.

[11] Kucuk, T. and Deveci, S. (2008) Chromohysteroscopy help target endometrial biopsy in postmenopausal bleeding. European Journal of Gynaecological Oncology, 29, 165-167. 